Article

\title{
Pinocembrin Protects the Brain against Ischemia-Reperfusion Injury and Reverses the Autophagy Dysfunction in the Penumbra Area
}

\author{
Gang Zhao, Wen Zhang, Li Li, Song Wu and Guanhua Du * \\ Beijing Key Laboratory of Drug Targets Identification and Drug Screening, Institute of Materia \\ Medica, Chinese Academy of Medical Sciences and Peking Union Medical College, Beijing 100050, \\ China; E-Mails: zhiweinuobeier@163.com (G.Z.); zhangwen9010@imm.ac.cn (W.Z.); \\ lili@imm.ac.cn (L.L.);ws@imm.ac.cn (S.W.) \\ * Author to whom correspondence should be addressed; E-Mail: dugh@imm.ac.cn; \\ Tel.: +86-10-6313-1571; Fax: +86-10-6316-5184.
}

External Editor: Derek J. McPhee

Received: 31 July 2014; in revised form: 6 September 2014 / Accepted: 15 September 2014/ Published: 30 September 2014

\begin{abstract}
The aim of this study was to investigate the effects of pinocembrin on brain ischemia/reperfusion ( $\mathrm{I} / \mathrm{R})$ injury and the potential involvement of autophagy activity changes in the penumbra area in the mechanisms of pinocembrin activity. Focal cerebral $\mathrm{I} / \mathrm{R}$ model was induced by middle cerebral artery occlusion (MCAO) for $2 \mathrm{~h}$ followed by $24 \mathrm{~h}$ reperfusion. Pinocembrin was administered intravenously at different doses $(1,3$, and $10 \mathrm{mg} / \mathrm{kg}$, respectively) at the onset of reperfusion. Neurological function, brain infarction and brain swelling ratio were evaluated. Terminal deoxynucleotidyl transferase-mediated dUTP nick end labeling (TUNEL) method and immunohistochemical analysis (Caspase-3) were used to evaluate apoptosis in the penumbra cortex. Two key proteins of autophagy, LC3B and Beclin1, were detected by western blot. The results showed that pinocembrin-treatment could significantly reduce neurological deficit scores, infarct volume, cerebral edema and improve pathological lesion in the I/R rats. Pinocembrin-treatment could also reduce the number of TUNEL-positive and Caspase-3-positive neurons, and upregulate the expression of LC3B and Beclin1 in the penumbra area. These results suggested that pinocembrin could protect the brain against I/R injury, and the possible mechanisms might be attributed to inhibition of apoptosis and reversed autophagy activity in the penumbra area.
\end{abstract}


Keywords: pinocembrin; ischemia/reperfusion; penumbra; autophagy

\section{Introduction}

Pinocembrin (5,7-dihydroxyflavanone), the most abundant flavonoid in propolis [1], has drawn much attention for its broad spectrum of pharmacological properties. Our previous studies have demonstrated that pinocembrin could protect the brain from ischemic injury $[2,3]$, and protection of neurovascular unit and mitochondria [4,5], anti-inflammation [5] and anti-oxidative stress [3] were proposed to be the possible mechanisms, but the real mechanisms underlying its protective effects and the exact targets are still being studied.

Ischemic cerebrovascular abnormality is a kind of severe health-threatening disorder, with high morbidity, mortality and possibility of causing permanent disability. Thrombolysis therapy plays a critical role in dealing with ischemic cerebrovascular abnormality. However, the blood reperfusion process after thrombolysis may cause a more serious tissue injury, namely $I / R$ injury [6,7]. It has been proposed that energy metabolism deficiency, oxidative stress [8], intracellular and mitochondrial calcium-overload [9], glutamate/neurotoxin release [10], inflammation [11], and apoptosis [12] may take part in the process of $\mathrm{I} / \mathrm{R}$ injury. Accumulating evidence indicates that autophagy also plays an important role in the mechanisms underlying I/R-induced neuronal damage [13-15].

Autophagy is a catabolic and conserved lysosomal degradation pathway that controls the quality of cytoplasm by eliminating the intracellular misfolded or aggregated proteins and damaged organelles. Mounting evidence has implicated the important roles of autophagy in several human diseases [16-21]. In the context of cerebral ischemia, some evidences indicated that autophagy might cause autophagic cell death [22], while some data suggested that autophagy might be neuroprotective [23,24], raising controversy with regard to the role of autophagy in ischemic brain injury. Given that autophagy might play an important role in I/R injury, the aim of this study was to investigate the potential involvement of autophagy activity change in the mechanisms of pinocembrin against I/R injury.

\section{Results and Discussion}

\subsection{Effects of Pinocembrin on Neurological Deficits Induced by $I / R$}

As shown in Figure 1A, MCAO for $2 \mathrm{~h}$, followed by $24 \mathrm{~h}$ of reperfusion induced obvious neurological deficit $(P<0.001)$. Pinocembrin decreased the neurological deficit scores in a dose-dependent manner $(P<0.001)$. The effect of pinocembrin, especially at the dose of $10 \mathrm{mg} / \mathrm{kg}$, on neurological deficit scores was even better than that of edaravone $(3.5 \mathrm{mg} / \mathrm{kg})$.

In addition, I/R could also significantly shorten the retention time of rats on the inclined plate $(P<0.001)$. Pinocembrin administration prolonged the retention time on the inclined plate in a dose-dependent manner $(P<0.001)$ and the effect was superior to edaravone, as illustrated in Figure 1B. 
Figure 1. Effects of pinocembrin on neurological deficits in the rats subjected to $2 \mathrm{~h}$ of MCAO followed by $24 \mathrm{~h}$ reperfusion. (A) Neurological deficit scores; (B) Retention time of rats on the inclined plate. Data are expressed as means \pm SD and analyzed by one-way ANOVA followed by Dunett's test. $\mathrm{n}=12 .{ }^{\# \#} P<0.001$ vs. I/R group, *** $P<0.01$ vs. sham group.

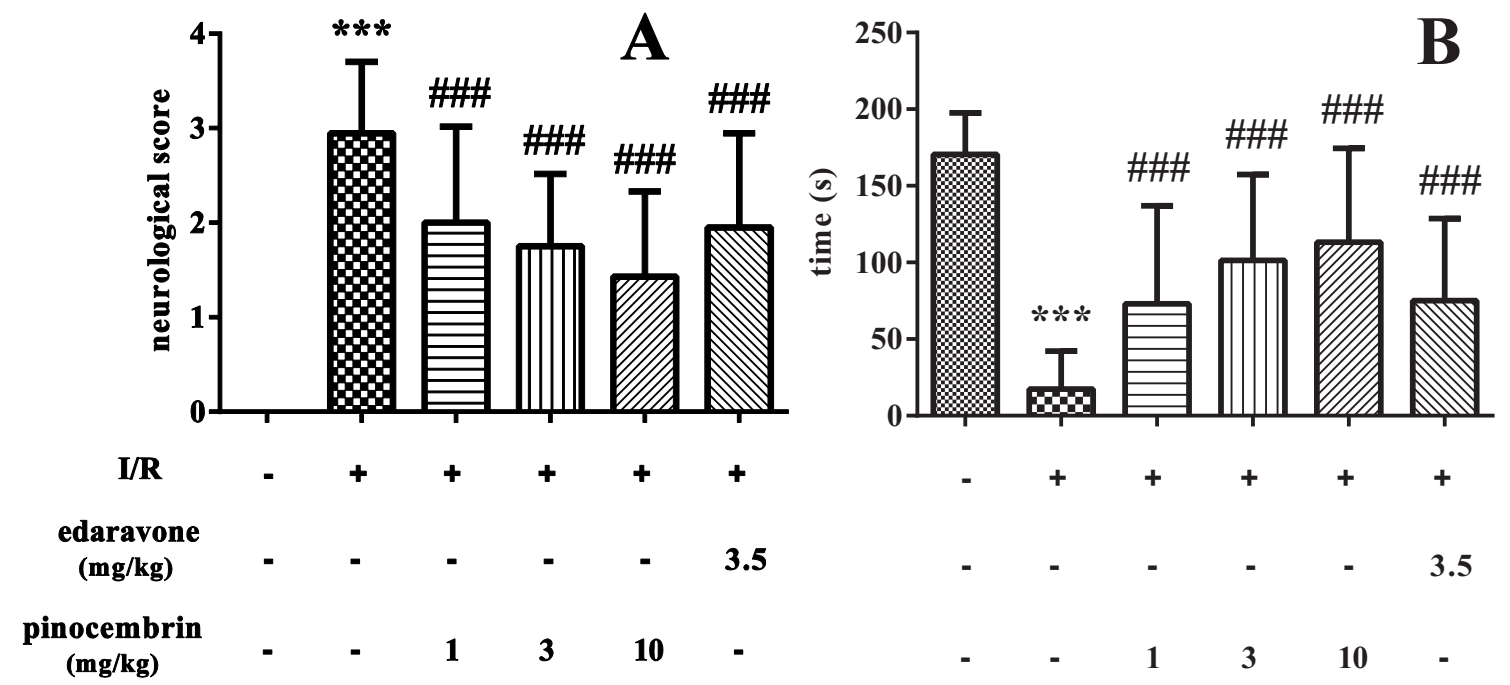

\subsection{Effects of Pinocembrin on Infarct Volume}

As illustrated in Figure 2, I/R induced severe infarct in the ischemic hemisphere $(51.28 \%$, $P<0.001)$. Pinocembrin significantly decreased the infarct volume in a dose-dependent manner. The effect of pinocembrin on infarct volume, especially at the dose of $10 \mathrm{mg} / \mathrm{kg}$, was even better than that of edaravone $(3.5 \mathrm{mg} / \mathrm{kg})$.

Figure 2. Effects of pinocembrin on brain infarct volume of rats subjected to $2 \mathrm{~h}$ of MCAO followed by $24 \mathrm{~h}$ reperfusion. (A) Representative TTC stained coronal sections showing infarct area in the ischemic cerebral hemisphere as distinct pale-stained area. (B) Effects of pinocembrin and edaravone on brain infarct volume. Data are expressed as means \pm SD and analyzed by one-way ANOVA followed by Dunett's test. $\mathrm{n}=12$. ${ }^{\#} P<0.01$, ${ }^{\# \#} P<0.001$ vs. I/R group, $* * * P<0.001$ vs. sham group.
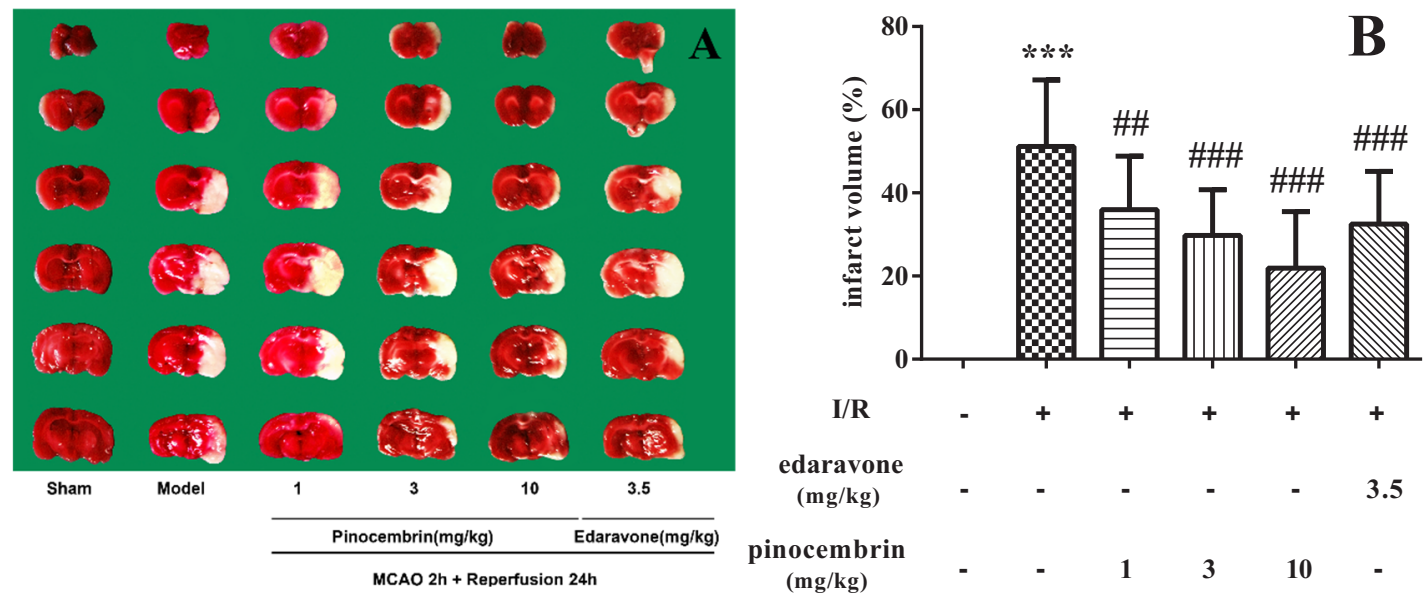


\subsection{Effects of Pinocembrin on Cerebral Edema Induced by $I / R$}

As shown in Figure 3, I/R induced severe cerebral edema $(22.91 \%, P<0.001)$. Pinocembrin could significantly ameliorate the cerebral edema in a dose-dependent manner. And at the dose of $10 \mathrm{mg} / \mathrm{kg}$, pinocembrin showed a better effect than edaravone did.

Figure 3. Effects of pinocembrin on brain swelling of rats subjected to $2 \mathrm{~h}$ of MCAO followed by $24 \mathrm{~h}$ reperfusion. Data are expressed as means $\pm \mathrm{SD}$ and analyzed by one-way ANOVA followed by Dunett's test. $\mathrm{n}=4-10$. ${ }^{\#} P<0.05,{ }^{\#} P<0.01$ vs. I/R group, $* * * P<0.001$ vs. sham group.

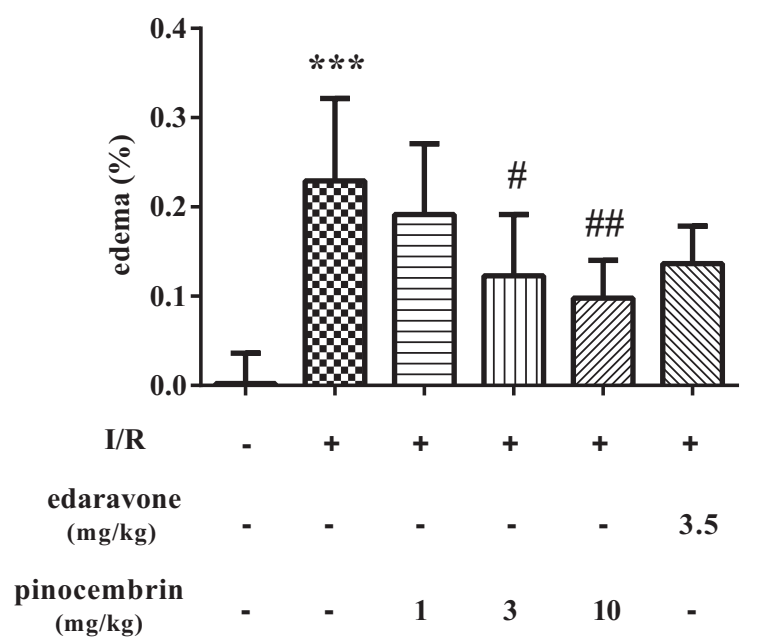

\subsection{Effects of Pinocembrin on Pathological Damages Induced by $I / R$}

\subsubsection{Effects of Pinocembrin on Pathological Damages in the Penumbra Area (Nissl's Staining)}

As illustrated in Figure 4B, I/R caused pathomorphological changes in the penumbra region. It could be seen that the neurons arranged loosely; the nucleus boundary of some neurons was not clear; nucleoli of some neurons disappeared; vacuole-like changes emerged; some Nissl bodies decreased; and cell gaps widened. Inflammatory cell infiltration could also be observed. Pinocembrin could effectively ameliorate the above-mentioned pathological damages, especially at the dose of $10 \mathrm{mg} / \mathrm{kg}$.

\subsubsection{Effects of Pinocembrin on Pathological Damages in Hippocampus CA1 Region (Nissl's Staining)}

As illustrated in Figure 5B. I/R caused serious pathological damages in the hippocampus CA1 region. It could be seen that the neurons arranged loosely; the number of neuron decreased obviously; the gaps around cells widened; the vacuole-like changes emerged; and some Nissl bodies decreased. Pinocembrin could effectively ameliorate the above-mentioned pathological damages, especially at the dose of $10 \mathrm{mg} / \mathrm{kg}$. 
Figure 4. Effects of pinocembrin on pathomorphological changes in the penumbra of rats subjected to $2 \mathrm{~h}$ of MCAO followed by $24 \mathrm{~h}$ reperfusion. Representative photographs of tissue sections stained with Nissl's staining method in the penumbra. The pathomorphological changes in model group were marked by a cycle (B). (A) sham; (B) model (I/R); (C) $\mathrm{I} / \mathrm{R}+$ pinocembrin $1 \mathrm{mg} / \mathrm{kg}$; (D) $\mathrm{I} / \mathrm{R}+$ pinocembrin $3 \mathrm{mg} / \mathrm{kg}$; (E) I/R + pinocembrin $10 \mathrm{mg} / \mathrm{kg}$; (F) edaravone $3.5 \mathrm{mg} / \mathrm{kg}$. (magnification: $200 \times$ ).
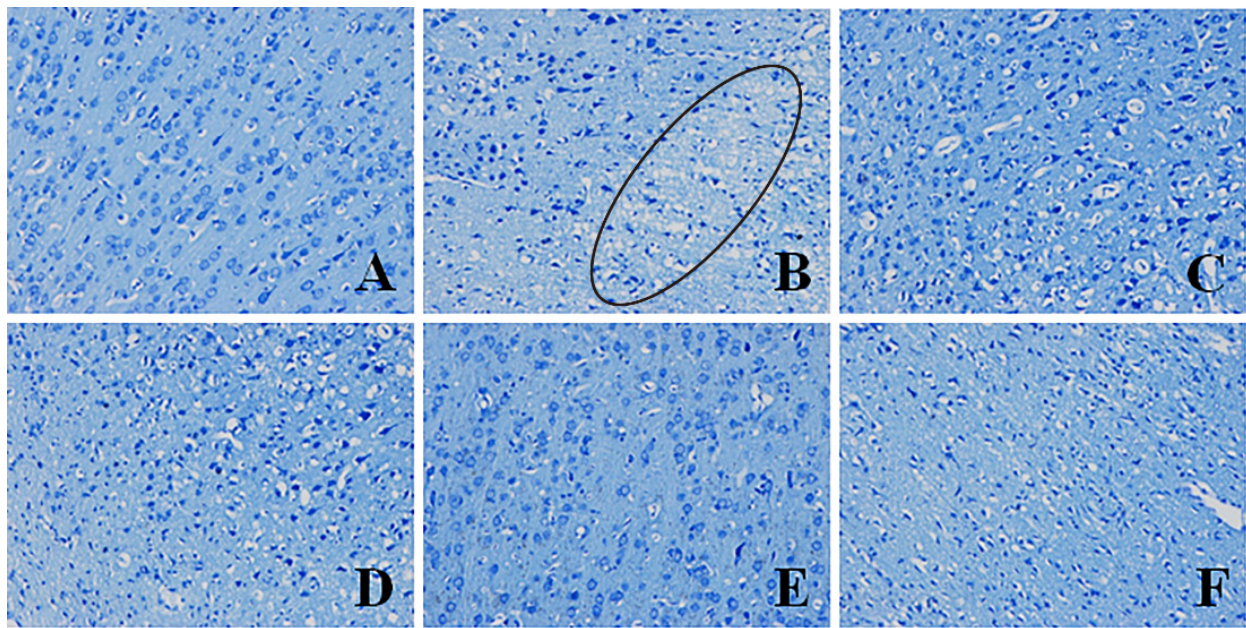

Figure 5. Effects of pinocembrin on pathomorphological changes in the hipocampus of rats subjected to $2 \mathrm{~h}$ of MCAO followed by $24 \mathrm{~h}$ reperfusion. Representative photographs of tissue sections stained with Nissl's staining method in the hipocampus. The pathomorphological changes in model group were marked by a cycle (B). (A) sham; (B) model (I/R); (C) I/R + pinocembrin $1 \mathrm{mg} / \mathrm{kg}$; (D) $\mathrm{I} / \mathrm{R}+$ pinocembrin $3 \mathrm{mg} / \mathrm{kg}$; (E) $\mathrm{I} / \mathrm{R}+$ pinocembrin $10 \mathrm{mg} / \mathrm{kg}$; (F) edaravone $3.5 \mathrm{mg} / \mathrm{kg}$. (magnification: $200 \times$ ).
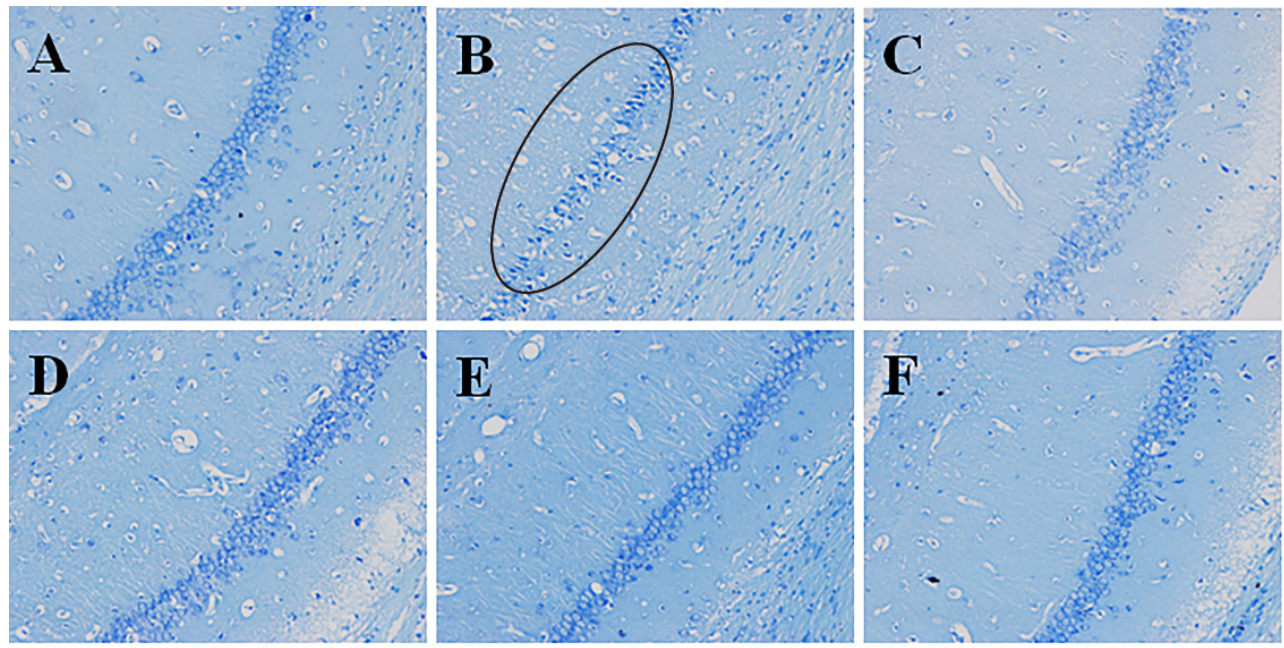

\subsection{Effects of Pinocembrin on the Expression of Caspase-3 in the Penumbra Area}

Caspase-3 plays an important role in regulating apoptosis. As illustrated in Figure 6, I/R significantly increased the expression of Caspase- 3 in the penumbra. Pinocembrin inhibited the upregulation of Caspase- 3 induced by $\mathrm{I} / \mathrm{R}$ in a dose-dependent manner. 
Figure 6. Effects of pinocembrin on Caspase-3 level in the penumbra area of rats subjected to $2 \mathrm{~h}$ of MCAO followed by $24 \mathrm{~h}$ reperfusion. (a) Representative photographs of tissue sections immunostained with Caspase-3 antibody in the penumbra area (magnification: 200×). A: sham; B: model (I/R); C: I/R + pinocembrin $1 \mathrm{mg} / \mathrm{kg} ; \mathbf{D}: \mathrm{I} / \mathrm{R}+$ pinocembrin 3 $\mathrm{mg} / \mathrm{kg}$; E: I $/ \mathrm{R}+$ pinocembrin $10 \mathrm{mg} / \mathrm{kg}$; F: edaravone $3.5 \mathrm{mg} / \mathrm{kg}$. (b) Quantification of the effect of pinocembrin on Caspase-3 positive cells number. Data are expressed as means \pm SD and analyzed by one-way ANOVA followed by Dunett's test. $(\mathrm{n}=3)$. ${ }^{\#} P<0.05,{ }^{\#} P<$ 0.01 vs. I/R group; $* * * P<0.001 v s$. sham group.

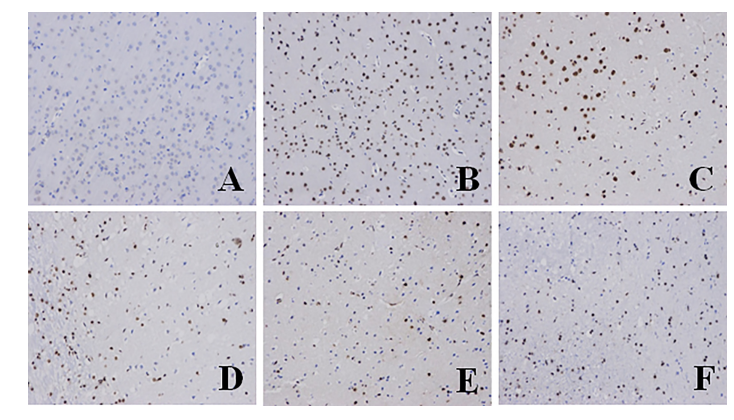

(a)

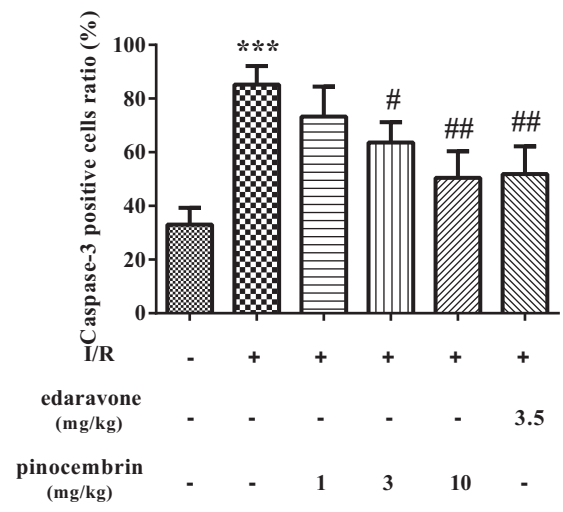

(b)

\subsection{Effects of Pinocembrin on Cell Apoptosis in the Penumbra Area}

As shown in Figure 7, virtually no TUNEL-positive cells were observed in sham group, while abundant TUNEL-positive cells were seen in I/R group. At the doses of 3 and $10 \mathrm{mg} / \mathrm{kg}$, pinocembrin could significantly decrease the number of TUNEL-positive cells.

Figure 7. Effects of pinocembrin on cell apoptosis in the penumbra cortex of the rats after subjected to $2 \mathrm{~h}$ of MCAO followed by $24 \mathrm{~h}$ reperfusion. (a) TUNEL staining was performed on the sections from the penumbra cortex (magnification: 200×). A: sham; B: $\mathrm{I} / \mathrm{R} ; \mathbf{C}: \mathrm{I} / \mathrm{R}+$ pinocembrin $1 \mathrm{mg} / \mathrm{kg} ; \mathbf{D}: \mathrm{I} / \mathrm{R}+$ pinocembrin $3 \mathrm{mg} / \mathrm{kg} ; \mathbf{E}: \mathrm{I} / \mathrm{R}+$ pinocembrin $10 \mathrm{mg} / \mathrm{kg}$; F: edaravone $3.5 \mathrm{mg} / \mathrm{kg}$. (b) Quantification of the effect of pinocembrin on ratio of tunel positive cells. Data are expressed as means \pm SD and analyzed by one-way ANOVA followed by Dunett's test. $\mathrm{n}=3$. ${ }^{\# \#} P<0.001$ vs. I/R group, *** $P<0.001$ vs. sham group.

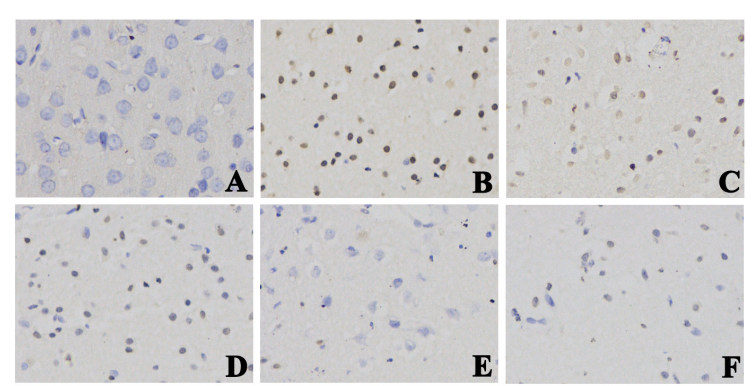

(a)

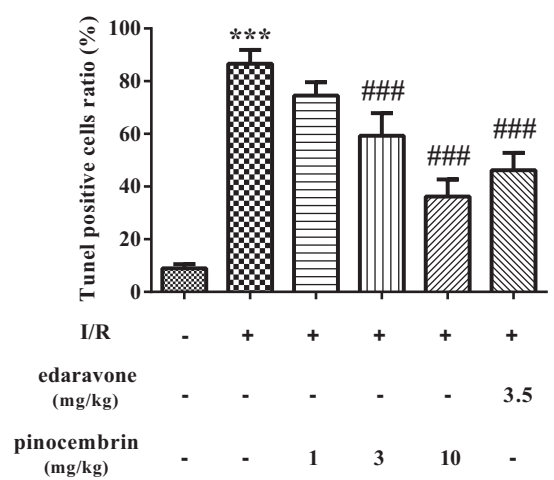

(b) 


\subsection{Effects of Pinocembrin on the Expression of Autophagy-Related Proteins in the Penumbra Area}

The expression of autophagy-related proteins was investigated by western blot. As shown in Figure 8 , in $\mathrm{I} / \mathrm{R}$ group, the levels of LC3B and Beclin1 in the penumbra decreased significantly. These results indicated that autophagy was retarded after $2 \mathrm{~h}$ of MCAO followed by $24 \mathrm{~h}$ reperfusion. Pinocembrin administration at the onset of reperfusion could significantly increase the levels of LC3B and Beclin1 in the penumbra after $24 \mathrm{~h}$ reperfusion. These data suggested that pinocembrin could reverse autophagy activity in the penumbra after $2 \mathrm{~h}$ of MCAO followed by $24 \mathrm{~h}$ reperfusion.

Figure 8. Effects of pinocembrin on autophagy-related protein level in the penumbra of rats subjected to $2 \mathrm{~h}$ of MCAO followed by $24 \mathrm{~h}$ reperfusion. (A) The expression of LC3B and Beclin1 was detected by western blot. (B) Quantitative analysis of the expression of LC3B and Beclin1. GAPDH served as loading control. Data are expressed as means \pm SD and analyzed by one-way ANOVA followed by Dunett's test $(\mathrm{n}=3)$. ${ }^{\#} P<0.05,{ }^{\#} P<0.01$ vs. I/R group, ${ }^{*} P<0.05, * * P<0.01$ vs. sham group.

B
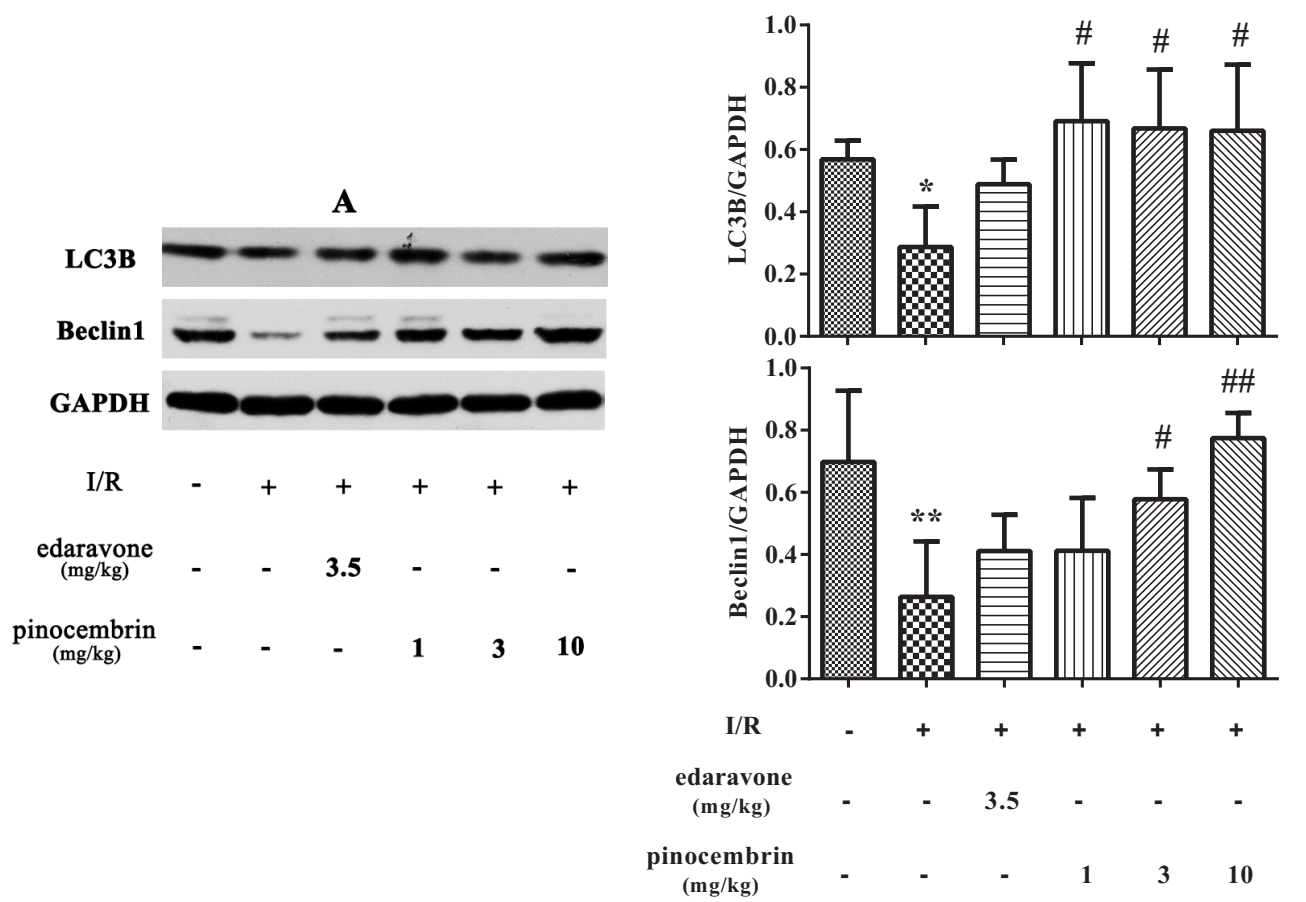

\subsection{Discussion}

In the present study, we confirmed that pinocembrin could protect the brain against $I / R$ injury, by ameliorating neurological deficits, decreasing the infarct volume and alleviating the cerebral edema, and exhibited better effects than edaravone did. In addition, we demonstrated that pinocembrin could improve the pathological lesion in the penumbra. Furthermore, we found that pinocembrin could inhibit apoptosis and reverse autophagy activity in the penumbra.

Ischemic stroke-caused cerebral damage can be divided by a core of necrotic cell death and penumbra, which surrounds the damage core [25]. In the penumbra area, the energy metabolism is 
preserved by constrained blood supply. Both basic investigators of cerebral ischemia and clinicians pay much attention on penumbra, because it is believed that the penumbra damage is reversible, while necrotic lesion in the damage core is irreversible. A large number of documents have suggested that apoptosis was activated in the penumbra [26,27]. In accordance with previous study, our results also indicated that I/R increased the numbers of Caspase-3 positive cells and TUNEL-positive cells in the penumbra, which partially demonstrated that apoptosis was activated. In addition, our results proved that pinocembrin could inhibit the upregulation of Caspase-3 and decrease the number of TUNEL-positive cells, which indicated that inhibition of apoptosis in the penumbra area may be one of the mechanisms by which pinocembrin protect the brain against $I / R$ injury.

The results of pathomorphological study also indicated that pinocembrin could protect the penumbra area. Based on these results, it is suggested that the protective effect on the penumbra may contribute to the therapeutic effect of pinocembrin on I/R injury.

In recent years, the importance of autophagy in ischemic disease has received much attention. Some researchers believe that inhibition of autophagy is neuroprotective and may be a novel strategy to prevent ischemic brain injury [28,29]. However, autophagy activation is also thought to be associated with neuroprotection in a rat model of focal cerebral ischemic preconditioning [23]. A recent study revealed that the cerebral I/R-induced autophagy protects against neuronal injury by mitochondrial clearance [24]. Our results support the view that autophagy plays a protective role in cerebral I/R. Microtubule-associated protein 1 light chain 3 (LC3) participates in the maturation process of aotophagosome. Beclin1 plays an important role in inducing autophagy. Our results indicated that the levels of LC3B and Beclin1 were decreased significantly in the penumbra after $24 \mathrm{~h}$ of reperfusion. These results suggested that autophagy activity in the penumbra was inhibited after $24 \mathrm{~h}$ of reperfusion. It seems that we came to a conclusion which was inaccordance with a previous study [24]. This distinction may be owing to the different criterion we applied in locating the penumbra area, different injury degree, and different surgery procedure we adopted in establishing I/R model. Our results also showed that pinocembrin administration at the onset of reperfusion could increase the levels of LC3B and Beclin1 in the penumbra after $24 \mathrm{~h}$ of reperfusion, which indicated that pinocembrin could reverse the autophagy activity in the penumbra, and we proposed that this effect may be involved in the mechanisms of pinocembrin against I/R injury. Further research is needed to elucidate how pinocembrin regulates the autophagy activity in the penumbra and to explore the potential targets within the autophagy pathway in order to establish new strategies to treat I/R injury.

\section{Experimental Section}

\subsection{Materials}

Male Sprague-Dawley rats (260-300 g) were obtained from the SPF Laboratory Animal Technology Co., Ltd. (Beijing, China) and housed at room temperature with a $12 \mathrm{~h}$ light/dark cycle and had access to standard rodent chow and fresh water ad libitum. All experiments were in complete compliance with the National Institutes of Health Guide for the Care and Use of Laboratory Animals. Efforts were made to minimize any pain or discomfort. Edaravone injection was set as positive control and purchased from Simcere (Nanjing, China). Pinocembrin for injection was provided by Department 
of Medical Synthetic Chemistry, Institute of Materia Medica, Chinese Academy of Medical Sciences. 2,3,5-Triphenyltetrazolium chloride (TTC) was purchased from Sigma Chemical Co. (Shanghai, China). Immunohistochemical assay kit (REALTMEnVision+/HRP RABBIT/MOUSE) was purchased from Dako Denmark A/S (Glostrup, Denmark).TUNEL staining kit was purchased from Hoffmann-La Roche Ltd. (Shanghai, China). The antibodies against LC3B, Beclin1 and Caspase-3 were purchased from Cell Signaling Technology, Inc. (Danvers, MA, USA). The suture used in MCAO was purchased from Beijing Cinontech Co. Ltd. (Beijing, China).

\subsection{Animal Model of MCAO and Drug Administration}

The male SD rats were randomly divided into six groups: sham operation group; I/R group; $\mathrm{I} / \mathrm{R}+$ pinocembrin $(1,3$ and $10 \mathrm{mg} / \mathrm{kg})$ group; $\mathrm{I} / \mathrm{R}+$ edaravone $(3.5 \mathrm{mg} / \mathrm{kg})$ group. Pinocembrin for injection was dissolved in $0.9 \%$ saline water. Focal cerebral I/R model was established by occlusion of the middle cerebral artery(MCAO) for $2 \mathrm{~h}$ followed by $24 \mathrm{~h}$ reperfusion according to previously described methods [30]. Briefly, the rats were anesthetized with $10 \%$ chloral hydrate (i.p. $380 \mathrm{mg} / \mathrm{kg}$ ). The right common carotid artery (CCA), external carotid artery (ECA) and internal carotid artery (ICA) were separated away from adjacent muscles and nerves. A fishing line coated with polylysine (diameter $0.32 \mathrm{~mm}$ ) was inserted from a mini-incision on the ECA and got to middle cerebral artery (MCA). After $2 \mathrm{~h}$ of ischemia, the filament was withdrawn to allow reperfusion for $24 \mathrm{~h}$. Pinocembrin and edaravone were administrated at the onset of reperfusion. After $12 \mathrm{~h}$ of reperfusion, pinocembrin and edaravone were administrated again.

\subsection{Neurological Deficit Score Assessment}

Neurological deficit score of each rat was assessed $24 \mathrm{~h}$ after reperfusion according to Bederson's method [31] with minor modification: 0, no neurological symptoms; 1, failure to extend left paw completely; 2, the strength of left fore-limb decreased obviously; 3 , rotating and crawling towards left side; 4, unable to walk spontaneously.

\subsection{Inclined Plane Test}

After $24 \mathrm{~h}$ of reperfusion, the rats were placed on the coarse surface of a inclined plane, the angle of which was set as $85^{\circ}$. The duration of each rat stayed on the inclined plane was recorded. For those rats which could stay on the inclined plane for more than $3 \mathrm{~min}$, the scores were recorded as $180 \mathrm{~s}$.

\subsection{Measurement of Cerebral Infarct Volume}

TTC staining method was applied for the measurement of cerebral infarct volume [32,33]. After the neurological deficit score assessment and inclined plane test, the rats were sacrificed, and the brains were quickly removed, frozen at $-20{ }^{\circ} \mathrm{C}$ for about $15 \mathrm{~min}$, and then sliced into six $2 \mathrm{~mm}$ thick coronal sections. The slices were stained with $0.5 \%$ TTC solution at $37{ }^{\circ} \mathrm{C}$ for $15 \mathrm{~min}$. The size of infarct area (unstained region shown pale) of each section was analyzed (Image-Pro Plus6.0, Media Cybernetics, Rockville, MD, USA). Infarction rate $(\%)=A^{\circ} / A^{\prime} \times 100 \%, A^{\circ}$ represented the infarct volume, $A^{\prime}$ was the volume of the homolateral hemisphere. 


\subsection{Measurement of Cerebral Edema}

After neurological function evaluation, the rats were decapitated, and the brains were quickly removed and the cerebellum and the brain stem were excised. And then the brains were dissected along the sagittal suture. Ischemic hemisphere and contralateral hemisphere were weighed. Cerebral edema $(\%)=\left(\mathrm{B}^{\circ}-\mathrm{B}^{\prime}\right) / \mathrm{B}^{\prime} \times 100 \%$, where $\mathrm{B}^{\circ}$ represented the weight of the ischemic hemisphere, $\mathrm{B}^{\prime}$ represented the homolateral hemisphere weight.

\subsection{Histopathological Assessment}

After neurological function evaluation, the rats were anesthetized with $10 \%$ chloral hydrate (i.p. $400 \mathrm{mg} / \mathrm{kg}$ ) and perfused with $4 \%$ paraformaldehyde in $0.1 \mathrm{M}$ phosphate buffer (PB, pH 7.4). The brains were removed and further fixed in $4 \%$ paraformaldehyde for $24 \mathrm{~h}$, embedded in paraffin wax. The paraffin-embedded blocks were cut into a series of $5 \mu \mathrm{m}$ thick slices which contained the penumbra area and stained with the Nissl's staining method. The penumbra area was located according to the principle described by Ashwal [34].

\subsection{Immunohistochemical Assay and Tunel Staining}

The expression of Caspase-3 in penumbra area was detected by immunohistochemical method, using a commercial kit, according to the manufacturer's instructions. Briefly, the paraffin-embedded brain sections were dewaxed and washed in PBS for three times, then immersed in $3 \%$ hydrogen peroxide for $3 \mathrm{~min}$ to block intrinsic peroxidase, quenched in BSA for $20 \mathrm{~min}$, and then the sections were reacted with a rabbit polyclonal antibody against Caspase-3 (1:200 dilutions) overnight at $4{ }^{\circ} \mathrm{C}$. Then, the sections were incubated with anti-rabbit secondary antibody for $50 \mathrm{~min}$ at $4{ }^{\circ} \mathrm{C}$. After that, 3,3'-diaminobenzidine (DAB) was used for colour development. Finally, haematoxylin was used for counterstaining. Image-Pro Plus6.0 software was used to analyze the Caspase-3 positive cells ratio. TUNEL staining for penumbra was performed using a commercial kit according to the manufacturer's instructions. Image-Pro Plus6.0 software was used to analyze the TUNEL positive cells ratio.

\subsection{Western Blot Analysis}

After the measurement of cerebral edema, the penumbra area was collected according to the principle described by Ashwal [34]. The total protein of penumbra was extracted. The concentration of the total protein was detected by Bradford method according to the manufacturer's instructions. An aliquot of $40 \mu \mathrm{g}$ protein from each sample was separated by SDS-PAGE gel electrophoresis and transferred onto a PVDF membrane (Millipore, Billerica, MA, USA), and then blocked with 5\% nonfat milk in $0.5 \%$ TBST for 1 hour. The transferred membranes were incubated overnight at $4{ }^{\circ} \mathrm{C}$ with primary antibodies against LC3B (1:1000 dilution), Beclin1 (1:1000 dilution). After being washed three times in TBST, the membranes were incubated with secondary antibodies against rabbit $(1: 1000$ dilution) IgG-horseradish peroxidase-conjugated for $0.5 \mathrm{~h}$ at room temperature. An enhanced chemiluminescence (ECL) was used to visualize the immunoreactive bands, which were captured on X-ray film. Optical density of the bands was analyzed by Quantity One software. 


\subsection{Statistical Analysis}

All data are expressed as means \pm SD. Differences between the groups were analyzed by one-way ANOVA followed by Dunett's test using the SPSS software 17.0. $P<0.05$ was considered statistically significant.

\section{Conclusions}

In conclusion, this study suggested that pinocembrin could protect the brain against ischemia-reperfusion injury, and inhibition of apoptosis and reversed autophagy activity in the penumbra area might be involved in the mechanisms.

\section{Acknowledgments}

This study was supported by National Scientific \& Technological Major Project for "Significant New Drugs Creation" (No. 2012ZX09103-101-078), Special Foundation for Scientific Research in Public Health Industry (No. 200902008), the National Natural Science Foundation of China (No. 81102444), and the Central Public Scientific Research Institution Fundamental Project (No. 2014CX05) by Chinese government.

\section{Author Contributions}

Guanhua Du designed the study and revised the paper; Song Wu synthesised and provided the pinocembrin; Gang Zhao and Wen Zhang performed the experiments; Gang Zhao analyzed the data and wrote the manuscript; $\mathrm{Li}$ Li performed part of the experiments and revised the paper.

\section{Conflicts of Interest}

The authors declare no conflict of interest.

\section{References}

1. Villanueva, V.R.; Barbier, M.; Gonnet, M.; Lavie, P. The flavonoids of propolis. Isolation of a new bacteriostatic substance: Pinocembrin (dihydroxy-5, 7 flavanone). Ann. Inst. Pasteur (Paris) 1970, 118, 84-87.

2. Meng, F.; Liu, R.; Gao, M.; Wang, Y.; Yu, X.; Xuan, Z.; Sun, J.; Yang, F.; Wu, C.; Du, G. Pinocembrin attenuates blood-brain barrier injury induced by global cerebral ischemia-reperfusion in rats. Brain Res. 2011, 1391, 93-101.

3. Shi, L.L.; Chen, B.N.; Gao, M.; Zhang, H.A.; Li, Y.J.; Wang, L.; Du, G.H. The characteristics of therapeutic effect of pinocembrin in transient global brain ischemia/reperfusion rats. Life Sci. 2011, 88, 521-528.

4. Gao, M.; Liu, R.; Zhu, S.Y.; Du, G.H. Acute neurovascular unit protective action of pinocembrin against permanent cerebral ischemia in rats. J. Asian Nat. Prod. Res. 2008, 10, 551-558. 
5. Gao, M.; Zhu, S.Y.; Tan, C.B.; Xu, B.; Zhang, W.C.; Du, G.H. Pinocembrin protects the neurovascular unit by reducing inflammation and extracellular proteolysis in MCAO rats. J. Asian Nat. Prod. Res. 2010, 12, 407-418.

6. Rosenthal, R.E.; Hamud, F.; Fiskum, G.; Varghese, P.J.; Sharpe, S. Cerebral ischemia and reperfusion: Prevention of brain mitochondrial injury by lidoflazine. J. Cereb. Blood Flow Metab. 1987, 7, 752-758.

7. Aronowski, J.; Strong, R.; Grotta, J.C. Reperfusion injury: Demonstration of brain damage produced by reperfusion after transient focal ischemia in rats. J. Cereb. Blood Flow Metab. 1997, 17, 1048-1056.

8. Murin, R.; Drgova, A.; Kaplan, P.; Dobrota, D.; Lehotsky, J. Ischemia/Reperfusion-induced oxidative stress causes structural changes of brain membrane proteins and lipids. Gen. Physiol. Biophys. 2001, 20, 431-438.

9. Katayama, Y.; Fukuchi, T.; McKee, A.; Terashi, A. Effect of nicardipine, a $\mathrm{Ca}^{2+}$ channel blocker, on pyruvate dehydrogenase activity and energy metabolites during cerebral ischemia and reperfusion in gerbil brain. Brain Res. 1998, 781, 212-217.

10. Culmsee, C.; Junker, V.; Wolz, P.; Semkova, I.; Krieglstein, J. Lubeluzole protects hippocampal neurons from excitotoxicity in vitro and reduces brain damage caused by ischemia. Eur. J. Pharmacol. 1998, 342, 193-201.

11. Xing, B.; Chen, H.; Zhang, M.; Zhao, D.; Jiang, R.; Liu, X.; Zhang, S. Ischemic post-conditioning protects brain and reduces inflammation in a rat model of focal cerebral ischemia/reperfusion. J. Neurochem. 2008, 105, 1737-1745.

12. Li, J.S.; Zhang, W.; Kang, Z.M.; Ding, S.J.; Liu, W.W.; Zhang, J.H.; Guan, Y.T.; Sun, X.J. Hyperbaric oxygen preconditioning reduces ischemia-reperfusion injury by inhibition of apoptosis via mitochondrial pathway in rat brain. Neuroscience 2009, 159, 1309-1315.

13. Carloni, S.; Buonocore, G.; Balduini, W. Protective role of autophagy in neonatal hypoxia-ischemia induced brain injury. Neurobiol. Dis. 2008, 32, 329-339.

14. Balduini, W.; Carloni, S.; Buonocore, G. Autophagy in hypoxia-ischemia induced brain injury: Evidence and speculations. Autophagy 2009, 5, 221-223.

15. Balduini, W.; Carloni, S.; Buonocore, G. Autophagy in hypoxia-ischemia induced brain injury. J. Matern. Fetal Neonatal Med. 2012, 25 (Suppl. 1), 30-34.

16. Shintani, T.; Klionsky, D.J. Autophagy in health and disease: A double-edged sword. Science 2004, 306, 990-995.

17. Martinet, W.; Agostinis, P.; Vanhoecke, B.; Dewaele, M.; de Meyer, G.R. Autophagy in disease: A double-edged sword with therapeutic potential. Clin. Sci. 2009, 116, 697-712.

18. Jiang, T.; Yu, J.T.; Zhu, X.C.; Wang, H.F.; Tan, M.S.; Cao, L.; Zhang, Q.Q.; Gao, L.; Shi, J.Q.; Zhang, Y.D.; et al. Acute metformin preconditioning confers neuroprotection against focal cerebral ischaemia by pre-activation of AMPK-dependent autophagy. Br. J. Pharmacol. 2014, 171, 3146-3157.

19. Boland, B.; Kumar, A.; Lee, S.; Platt, F.M.; Wegiel, J.; Yu, W.H.; Nixon, R.A. Autophagy induction and autophagosome clearance in neurons: Relationship to autophagic pathology in Alzheimer's disease. J. Neurosci. 2008, 28, 6926-6937.

20. Yang, A.; Kimmelman, A.C. Inhibition of autophagy attenuates pancreatic cancer growth independent of TP53/TRP53 status. Autophagy 2014, 10, 1683-1684. 
21. Margaritopoulos, G.A.; Tsitoura, E.; Tzanakis, N.; Spandidos, D.A.; Siafakas, N.M.; Sourvinos, G.; Antoniou, K.M. Self-eating: Friend or foe? The emerging role of autophagy in idiopathic pulmonary fibrosis. BioMed Res. Int. 2013, 2013, doi:10.1155/2013/420497.

22. Wen, Y.D.; Sheng, R.; Zhang, L.S.; Han, R.; Zhang, X.; Zhang, X.D.; Han, F.; Fukunaga, K.; Qin, Z.H. Neuronal injury in rat model of permanent focal cerebral ischemia is associated with activation of autophagic and lysosomal pathways. Autophagy 2008, 4, 762-769.

23. Sheng, R.; Zhang, L.S.; Han, R.; Liu, X.Q.; Gao, B.; Qin, Z.H. Autophagy activation is associated with neuroprotection in a rat model of focal cerebral ischemic preconditioning. Autophagy 2010 , 6, 482-494.

24. Zhang, X.; Yan, H.; Yuan, Y.; Gao, J.; Shen, Z.; Cheng, Y.; Shen, Y.; Wang, R.R.; Wang, X.; $\mathrm{Hu}, \mathrm{W} . \mathrm{W}$; et al. Cerebral ischemia-reperfusion-induced autophagy protects against neuronal injury by mitochondrial clearance. Autophagy 2013, 9, 1321-1333.

25. Hossmann, K.A. Viability thresholds and the penumbra of focal ischemia. Ann. Neurol. 1994, 36, $557-565$.

26. Yuan, J. Neuroprotective strategies targeting apoptotic and necrotic cell death for stroke. Apoptosis 2009, 14, 469-477.

27. Graham, S.H.; Chen, J. Programmed cell death in cerebral ischemia. J. Cereb. Blood Flow Metab. 2001, 21, 99-109.

28. Xing, S.; Zhang, Y.; Li, J.; Zhang, J.; Li, Y.; Dang, C.; Li, C.; Fan, Y.; Yu, J.; Pei, Z.; et al. Beclin 1 knockdown inhibits autophagic activation and prevents the secondary neurodegenerative damage in the ipsilateral thalamus following focal cerebral infarction. Autophagy 2012, 8, 63-76.

29. Puyal, J.; Vaslin, A.; Mottier, V.; Clarke, P.G. Postischemic treatment of neonatal cerebral ischemia should target autophagy. Ann. Neurol. 2009, 66, 378-389.

30. Longa, E.Z.; Weinstein, P.R.; Carlson, S.; Cummins, R. Reversible middle cerebral artery occlusion without craniectomy in rats. Stroke 1989, 20, 84-91.

31. Bederson, J.B.; Pitts, L.H.; Tsuji, M.; Nishimura, M.C.; Davis, R.L.; Bartkowski, H. Rat middle cerebral artery occlusion: Evaluation of the model and development of a neurologic examination. Stroke 1986, 17, 472-476.

32. Bederson, J.B.; Pitts, L.H.; Germano, S.M.; Nishimura, M.C.; Davis, R.L.; Bartkowski, H.M. Evaluation of 2,3,5-triphenyltetrazolium chloride as a stain for detection and quantification of experimental cerebral infarction in rats. Stroke 1986, 17, 1304-1308.

33. Li, F.; Irie, K.; Anwer, M.S.; Fisher, M. Delayed triphenyltetrazolium chloride staining remains useful for evaluating cerebral infarct volume in a rat stroke model. J. Cereb. Blood Flow Metab. 1997, 17, 1132-1135.

34. Ashwal, S.; Tone, B.; Tian, H.R.; Cole, D.J.; Pearce, W.J. Core and penumbral nitric oxide synthase activity during cerebral ischemia and reperfusion. Stroke 1998, 29, 1037-1046.

Sample Availability: Samples of pinocembrin for injection is available from the authors.

(C) 2014 by the authors; licensee MDPI, Basel, Switzerland. This article is an open access article distributed under the terms and conditions of the Creative Commons Attribution license (http://creativecommons.org/licenses/by/4.0/). 